\title{
Prevalência do anticorpo da hepatite A em crianças $e$ adolescentes com hepatopatia crônica
}

\author{
Prevalence of hepatitis A antibody in children and adolescents with chronic liver disease
}

\author{
Cristina T. Ferreira ${ }^{1}$, Adriano N. R. Taniguchi ${ }^{2}$, Sandra M. Vieira ${ }^{3}$, \\ Jorge P. Lima ${ }^{4}$, Themis R. da Silveira ${ }^{5}$
}

\section{Resumo}

Objetivo: avaliar a prevalência de hepatite viral A (HVA) em crianças e adolescentes portadores de doenças crônicas do fígado, em um serviço de hepatologia pediátrica.

Métodos: entre maio de 1999 e fevereiro de 2001, foi estudada a prevalência de anticorpos anti-HVA total em 60 crianças e adolescentes, entre 1 e 16 anos de idade, portadoras de hepatopatias crônicas, provenientes da unidade de gastroenterologia pediátrica e programa de transplante hepático infantil do serviço de pediatria do Hospital de Clínicas de Porto Alegre. O anti-HVA, realizado através de um teste laboratorial comercialmente disponível em nosso meio (Abbott - MEIA HAVAB - sistema AXSYM), foi determinado e relacionado com a idade, com o sexo, com a cor, com o diagnóstico etiológico da hepatopatia e com a renda familiar dos pacientes.

Resultados: apenas uma criança de 1 ano, portadora de atresia biliar, foi excluída do estudo por apresentar anti-HVA indeterminado, em duas ocasiões. Das 59 crianças restantes, 14 (24\%) apresentavam resultados positivos para o anti-HVA total. As idades dos pacientes com anti-HVA positivos variaram de 1 a 16 anos $(x=7,7$ anos e mediana 8,5 anos). Não houve diferença significante entre idade, sexo e cor entre os grupos positivo e negativo. A renda familiar foi menor no grupo dos pacientes anti-HVA positivo, mas não mostrou diferença estatística significante. A diferença de prevalência de anti-HVA entre as etiologias das hepatopatias está, provavelmente, relacionada à idade mais do que ao diagnóstico.

Conclusões: na população estudada, a maioria (76\%) das crianças e adolescentes portadoras de hepatopatias crônicas é suscetível à infecção pelo vírus $\mathrm{A}$, podendo apresentar, portanto, um curso mais grave e complicações, se adquirirem HVA. Sugerimos, então, que esses pacientes devam receber a vacina inativada contra HVA.

J Pediatr (Rio J) 2002;78(6):503-8: hepatite viral A, prevalência, anticorpo anti-HVA, hepatopatias crônicas.

\begin{abstract}
Objective: to evaluate the prevalence of Hepatitis A virus (HAV) in children and adolescents with chronic liver disease in a unit of pediatric hepatology.

Methods: between May 1999 and February 2001, we studied the prevalence of anti-HAV in 60 children and adolescents with chronic liver disease, aged between 1 and 16 years, from the Unit of Pediatric Hepatology of the Hospital de Clínicas de Porto Alegre. The total anti-HAV was determined by a commercially available competitive ELISA method (Abbott), and compared with age, sex, race, etiologic diagnosis and family income of each patient.
\end{abstract}

Results: a one-year old child was not included in the study because she presented twice with undetermined anti-HAV results. Among the other 59 patients, 14 (24\%) presented a positive result of total anti-HAV. The ages of test-positive subjects varied between 1 and 16 years old (mean $=7.7$ years, median $=8.5$ ). The differences between positive and negative groups in relation to age, sex and race were not statistically significant. Family income was lower in antiHAV positive patients, but this difference was not significant. The differences between the etiologies of liver diseases were probably more related to the age than to the etiologies of the diseases.

Conclusions: in the studied population, the majority (76\%) of children and adolescents with chronic liver disease are susceptible to hepatitis A virus infection and, consequently, they could present a more severe disease or even fulminant hepatitis A. We strongly suggest that these subjects receive Hepatitis A inactivated vaccine.

JPediatr(Rio J) 2002;78(6):503-8: viral hepatitis A, prevalence, anti-HAV antibody, chronic liver disease.

1. Gastroenterologista e endoscopista pediátrica do Hospital de Clínicas de Porto Alegre (HCPA) e da Santa Casa de Misericórdia de Porto Alegre.

2. Acadêmico de Medicina da Universidade Federal do Rio Grande do Sul (UFRGS).

3. Gastroenterologista pediátrica do HCPA.

4. Professor adjunto do Pós-Graduação de Hepatologia da Fundação Faculdade Federal de Ciências Médicas de Porto Alegre (FFFCMPA); livre-docente de Medicina Interna da FFFCMPA.

5. Professora adjunta, Dra. do Departamento de Pediatria da Faculdade de Medicina da UFRGS.

Artigo submetido em 18.02.02, aceito em 28.08.02. 


\section{Introdução}

Hepatite viral aguda A (HVA) é uma infecção que existe em todo o mundo, e que é responsável por significativa morbidade. Do ponto de vista epidemiológico, a disseminação rápida e os grandes surtos de HVA, além da ausência de evolução para a cronicidade, a distinguem das Hepatites B e $\mathrm{C}^{1}$. A infecção pelo vírus A pode também causar hepatite fulminante e ser fatal, com uma taxa estimada de mortalidade de 0,14 a $2 \%^{2}$.

Há crescentes evidências na literatura médica de que indivíduos com doença hepática crônica apresentam um risco aumentado de desenvolver formas mais graves de HVA, ou mesmo hepatite fulminante ${ }^{1,3}$.

Os receptores de transplante de fígado devem evitar lesões adicionais no enxerto, devendo ser imunizados contra HVA, mesmo se o risco de exposição for baixo ${ }^{4}$. Além disso, os hepatopatas crônicos, que necessitarão um transplante de fígado, devem ser submetidos a todas as vacinações antes do procedimento cirúrgico, já que, posteriormente, ficarão mais imunocomprometidos que antes do transplante. Por esse motivo, esse grupo específico de pacientes tornou-se uma indicação de vacinação contra hepatite viral $\mathrm{A}^{4-6}$.

As crianças portadoras de hepatopatias crônicas teoricamente se beneficiariam com a vacina contra HVA, já que esta se mostrou imunogênica e segura em trabalhos controlados com crianças imunocompetentes ${ }^{6-8}$. $O$ fato de que a vacina é segura e eficaz em crianças e adultos saudáveis não garante resultados similares em populações com hepatopatia crônica ${ }^{1}$. Estudos realizados em adultos sugerem que, embora os hepatopatas crônicos não estejam sob um risco aumentado para infecção, eles apresentam um maior risco de hepatite fulminante pelo vírus $\mathrm{A}^{2,9,20}$. Os registros de óbito americanos indicam uma maior prevalência de doença hepática crônica entre pessoas que morreram de HVA fulminante do que entre pessoas que morreram por outras causas $5,6,9,10$.

Ainda não foi definido o esquema de imunização e a taxa de soroconversão para populações com doenças hepáticas crônicas ${ }^{1}$.

O objetivo deste estudo foi avaliar a prevalência de HVA em crianças e adolescentes portadores de doenças crônicas do fígado, em um serviço de hepatologia pediátrica.

\section{Pacientes e métodos}

No período de maio de 1999 a fevereiro de 2001, foram estudadas 60 crianças e adolescentes, portadoras de hepatopatias crônicas, provenientes da unidade de gastroenterologia pediátrica e programa de transplante hepático infantil do Serviço de Pediatria do Hospital de Clínicas de Porto Alegre. Este é um hospital terciário, referência em hepatologia pediátrica.
Foram incluídos no estudo pacientes com hepatopatias crônicas, com idades compreendidas entre 1 e 16 anos, sem história prévia de hepatite A, ou vacinação contra HVA, que não fossem portadores do vírus da imunodeficiência humana (HIV). Todos eram pacientes em avaliação, ou já avaliados para transplante de fígado, sendo que alguns estavam em lista de espera e outros não tinham indicação no momento da avaliação. Os pacientes eram portadores de doenças crônicas do fígado com confirmação histológica, através de biópsia hepática, ou ultra-sonografia e exames laboratoriais.

Foi um estudo transversal em que se avaliou a presença do anticorpo anti-HVA total, que indica infecção pelo vírus da hepatite A. O anti-HVA total foi determinado e relacionado com a idade, com o sexo, com a cor, com o diagnóstico etiológico da hepatopatia e com a renda familiar dos pacientes.

As idades variaram de 12 meses a 16 anos, com uma média ( \pm DP) de 7 anos $( \pm 4,9)$. Havia 25 crianças entre 1 e 4 anos, 9 entre 5 e 8 anos, 19 entre 9 e 12 , e 7 adolescentes entre 13 e 16 anos de idade.

Trinta e três crianças (55\%) eram do sexo feminino, e 92\% eram brancos.

Os diagnósticos etiológicos das hepatopatias encontram-se na Tabela 1.

A média de idade dos pacientes portadores de atresia de vias biliares extra-hepáticas (AVBEH) era 3,9 anos, a dos pacientes do grupo auto-imune era 11,8 anos, dos com cirrose criptogênica era de 6,2 anos, e dos outros era de 9 anos.

A renda familiar desses pacientes variou de 150,00 a $3.000,00$ reais, com uma média de 595,50 reais. A renda familiar de $73 \%$ dos pacientes era menor ou igual a 500,00 reais, e de $63 \%$ era de até $\mathrm{R} \$ 300,00$. O salário mínimo nessa ocasião era de $\mathrm{R} \$ 136,00$. A mediana da renda familiar foi de 300 reais, com variações interquartil de $\mathrm{R} \$$ 200,00 (percentil 25) a $\mathrm{R} \$ 675,00$ (p-75).

Tabela 1- Diagnósticos etiológicos das hepatopatias crônicas

\begin{tabular}{lcc}
\hline Diagnósticos & N & $(\%)$ \\
\hline AVBEH & 26 & $(43)$ \\
Hepatite auto-imune & 13 & $(22)$ \\
Cirrose criptogênica & 11 & $(18)$ \\
Fibrose cística & 2 & $(3)$ \\
Hepatite B & 2 & $(3)$ \\
Hepatite C & 1 & $(2)$ \\
Histiocitose & 1 & $(2)$ \\
Colangite esclerosante & 1 & $(2)$ \\
Fibrose hepática congênita & 1 & $(2)$ \\
Budd-Chiari & 1 & $(2)$ \\
Alagille & 1 & $(2)$
\end{tabular}

$\mathrm{AVBEH}=$ atresia de vias biliares extra-hepáticas 
O anti-HVA foi realizado através de um teste laboratorial, comercialmente disponível em nosso meio (Abbott MEIA HAVAB - sistema AXSYM). O paciente era submetido a uma coleta de sangue venoso, em geral no mesmo momento de suas coletas de rotina, que era centrifugado e congelado, para posterior avaliação. Os testes anti-HVA total foram todos realizados no mesmo laboratório de análises clínicas (Laboratório Weinmann), em Porto Alegre. Os resultados foram considerados positivos, negativos ou indeterminados de acordo com controles positivos e negativos. Calculou-se o valor de corte, abaixo do qual a amostra era positiva, e acima, negativa. Os testes com absorbância dentro da zona cinza, que corresponde a mais ou menos $10 \%$ do valor de corte, foram considerados indeterminados. Esses eram repetidos, em uma nova amostra, e se continuassem na zona cinza, ou seja, indeterminados, eram excluídos do estudo.

Os dois grupos (anti-HVA positivo e negativo) foram comparados utilizando teste do qui-quadrado com correlação de Yates (para variável sexo), teste exato de Fisher (para cor), teste $t$ de student (para idade) e teste U de MannWhitney (para renda familiar). Além disso, foi utilizado o teste do qui-quadrado para tendência linear, para verificar associação entre positividade do anti-HVA e aumento da idade dos pacientes. O nível de significância usado foi de 0,05 .

O protocolo de pesquisa foi aprovado pelo Comitê de Ética e Pesquisa do Hospital de Clínicas de Porto Alegre. Todos os pais dos pacientes ou responsáveis legais pelas crianças assinaram o consentimento escrito após informação do que se tratava o estudo.

\section{Resultados}

Das 60 crianças e adolescentes portadores de hepatopatia crônica, apenas uma foi excluída porque apresentou, em duas ocasiões diferentes, anticorpo anti-HVA indeterminado. Uma outra menina, de 1 ano de idade, também apresentou o primeiro teste indeterminado, mas negativou quando repetido 2 semanas depois.

Das 59 crianças e adolescentes que permaneceram no estudo, 14 (24\%) apresentaram resultados positivos para o anti-HVA total. As idades dos pacientes com anti-HVA positivos variaram de 1 a 16 anos ( $x=7,7$ anos e mediana 8,5 anos). Houve uma tendência da percentagem de positividade aumentar com a idade, embora não tenha havido uma comprovação estatística $\left(\chi^{2}\right.$ para tendência linear $\left.=0,6\right)$ (Tabela 2). A comparação entre o grupo dos positivos com o dos negativos está na Tabela 3.

Das crianças anti-HVA positivas, 5 eram portadores de hepatopatia crônica auto-imune, 4 de cirrose criptogênica, 3 de AVBEH, 1 de Budd-Chiari, e 1 de cirrose biliar associada à histiocitose.

Tabela 2 - Positividade do anti-HVA em relação à idade

\begin{tabular}{lccc}
\hline Faixa etária & $\begin{array}{c}\text { Anti-HVA } \\
\text { positivos (n) }\end{array}$ & $\begin{array}{c}\text { Total } \\
(\mathbf{n})\end{array}$ & $\begin{array}{c}\text { Percentagem de } \\
\text { positividade * }\end{array}$ \\
\hline 1 a 4 anos & 5 & 25 & $20 \%$ \\
5 a 8 anos & 2 & 9 & $22 \%$ \\
9 a 12 anos & 5 & 19 & $26 \%$ \\
13 a 16 anos & 2 & 7 & $29 \%$ \\
\hline
\end{tabular}

*Qui-quadrado para tendência linear $p=0,6$

Tabela 3 - Características dos pacientes (anti-HVA positivos e negativos)

\begin{tabular}{|c|c|c|c|c|}
\hline Característica & Total $(n=60)$ & Positivos $(n=14)$ & Negativos $(n=45)$ & Valor de $p$ \\
\hline \multicolumn{5}{|l|}{ Idade (anos) } \\
\hline Média & 7 & 7,7 & 6,76 & $0,59 *$ \\
\hline Desvio-padrão & 4,93 & 4,94 & 4,97 & \\
\hline Mediana & 7 & 8,5 & 6,5 & \\
\hline \multicolumn{5}{|l|}{ Cor } \\
\hline Branca $\mathrm{n}^{\circ}(\%)$ & $55(92)$ & $13(93)$ & $42(93)$ & $1,0^{\dagger}$ \\
\hline \multicolumn{5}{|l|}{ Sexo } \\
\hline Masculino $\mathrm{n}^{\circ}(\%)$ & $27(45)$ & $7(50)$ & $20(44)$ & $0,95^{\ddagger}$ \\
\hline $\begin{array}{l}\text { Renda familiar } \\
\text { (Reais - variação) }\end{array}$ & $150-3.000$ & $150-2.000$ & $150-3.000$ & \\
\hline Média & 595,50 & 441,00 & 642,61 & \\
\hline Desvio-padrão & 676,49 & 498,74 & 720,03 & \\
\hline Mediana & 300,00 & 300,00 & 300,00 & $0,26^{\text {I }}$ \\
\hline$\leq 500 \mathrm{n}^{\circ}(\%)$ & $44(73)$ & $12(86)$ & $32(71)$ & \\
\hline$>500 \leq 1.000 \mathrm{n}^{\circ}(\%)$ & $8(13)$ & $1(7)$ & $7(15,5)$ & \\
\hline$>1.000 \mathrm{n}^{\circ}(\%)$ & $8(13)$ & $1(7)$ & $7(15,5)$ & \\
\hline
\end{tabular}

* Teste $t$ de Student; ${ }^{\dagger}$ teste exato de Fisher; ${ }^{\ddagger}$ qui-quadrado com correção de Yates; ^ Mann Whitney 
A renda familiar do grupo de pacientes anti-HVA positivo ficou entre 150,00 e $2.000,00$ reais, sendo a média 441,00 reais, um pouco mais baixa do que a média do grupo dos 45 pacientes anti-HVA negativos ( $\mathrm{R} \$ 642,61)$. Doze $(86 \%)$ pacientes anti-HVA positivo apresentavam renda familiar menor do que 500,00 reais, enquanto que $69 \%$ dos negativos apresentavam-se nessa faixa. Onze crianças (79\%), entre os anti-HVA positivos, tinham renda familiar menor do que $\mathrm{R} \$ 300,00$. A mediana da renda familiar dos pacientes anti-HVA positivos foi de $\mathrm{R} \$ 300,00$ (p-25 R \$ 150,00 - p-75 R \$350,00).

Dos 5 pacientes anti-HVA positivos, portadores de hepatopatia auto-imune, as idades variaram entre 7 e 13 anos, com média de 11 anos e mediana de 12 anos. Todos eram meninas, e uma era de cor preta. A renda familiar média desse grupo foi de $\mathrm{R} \$ 380,00$, e a mediana, de $\mathrm{R} \$ 300,00$. Dois dos 4 pacientes positivos, portadores de cirrose criptogênica, eram do sexo masculino. As idades eram 1, 3, 6 e 10 anos, com uma média de 5 anos. A renda familiar variou de $\mathrm{R} \$ 150,00$ a $\mathrm{R} \$ 500,00$, com média de $\mathrm{R} \$ 267,50$. Os 3 pacientes anti-HVA positivos, com AVBEH, tinham 1, 2 e 16 anos de idade, respectivamente. Todos eram meninos e brancos. As rendas familiares eram de $\mathrm{R} \$ 300,00$ para 2 deles, e de $\mathrm{R} \$ 2.000,00$ para o outro (Tabela 4).

\section{Discussão}

Diferentes padrões de prevalência do anticorpo antiHVA têm sido descritos, com variações que refletem o nível de desenvolvimento econômico ${ }^{11}$. Em áreas de alta endemicidade, $90 \%$ das crianças são infectadas por volta dos 10 anos de idade. As infecções são assintomáticas, e a hepatite viral A não é um problema clínico. Nas áreas de endemicidade intermediária, as taxas de soroprevalência de $90 \%$ não são atingidas até o início da idade adulta. Nessas comunidades, a doença ocorre em faixas etárias variáveis (crianças, adolescentes e adultos jovens). Essas populações apresentam grandes epidemias, em intervalos regulares, que persistem por longos períodos de tempo, ou têm taxas elevadas, sustentadas de doença, por muitos anos $6,12,13$.

Embora seja conveniente pensar nesses diferentes padrões como mutuamente exclusivos, eles simplificam uma epidemiologia muito complexa. Em muitos países, os três padrões podem ocorrer entre diferentes grupos de uma mesma comunidade ${ }^{14}$. Então, esses padrões gerais de endemicidade podem variar dentro de um mesmo país, de uma mesma cidade, ou de mesmas regiões. No ano de 1994, estudamos a prevalência de HVA em 387 crianças e adolescentes, de 1 a 19 anos de idade, de Porto Alegre ${ }^{13}$. Encontramos, nesse primeiro estudo, uma diferença muito significativa de prevalência entre as crianças de baixo nível socioeconômico e as de alto nível ( $51 \%$ x $11 \%)$. Isso mostra que, ao mesmo tempo que a HVA é endêmica em nosso meio, existe um número significativo de pessoas suscetíveis à infecção, 89\% das crianças até os 19 anos, de alto nível socioeconômico, e $49 \%$ daquelas que vivem em condições econômicas mais desfavorecidas ${ }^{13}$. Nesse mesmo estudo, a prevalência de anti-HVA foi de $60 \%$ e de $11 \%$ até os 14 anos, nas classes de baixo e alto nível socioeconômico, respectivamente. Existem outros estudos no Brasil que mostram diferentes taxas de prevalência, dependendo do lugar estudado, mas todas mais altas do que as encontradas no Rio Grande do Sul ${ }^{15}$. No presente estudo, a prevalência de anti-HVA foi de $24 \%$ até os 16 anos, portanto mais baixa que no estudo anterior, provavelmente por diferenças de nível socioeconômico dos indivíduos estudados. Como os critérios de avaliação de nível socioeconômico empregados nos dois estudos foram distintos, a comparação tornase difícil.

A HVA, superposta à doença hepática crônica, tem sido associada com cursos mais graves, ou com formas fulminantes $1,2,9,10,16,17$. É possível que o insulto agudo imposto pela hepatite viral A possa causar alteração substancial da função hepática, previamente comprometida por lesão crônica. A exata incidência de insuficiência hepática por HVA em doença crônica do fígado não é conhecida ${ }^{17}$.

Tabela 4 - Idade e renda familiar dos pacientes de acordo com a etiologia da hepatopatia crônica

\begin{tabular}{llll}
\hline Grupos & Total (60) & Positivo (14) & Negativo (45) \\
\hline Atresia de vias biliares & 26 & $3(12 \%)$ & $22(88 \%)$ \\
Média idade (anos) & 3,9 & 6,3 & 3,56 \\
Média renda familiar (reais) & 578,46 & 866,66 & 540,87 \\
Hepatite auto-imune & 13 & $5(38,5 \%)$ & $8(61,5 \%)$ \\
Média idade (anos) & 11,8 & 11 & 12,3 \\
Média renda familiar (reais) & 483,84 & 380,00 & 548,75 \\
Criptogênica & 11 & $4(36 \%)$ & $7(64 \%)$ \\
Média idade (anos) & 6,2 & 5 & 7 \\
Média renda familiar (reais) & 309,09 & 267,50 & 332,85 \\
Outros & 10 & $2(20 \%)$ & $8(80 \%)$ \\
Média idade (anos) & 9 & 7 & 9,5 \\
Média renda familiar (reais) & 830,00 & 300,00 & 962,00 \\
\hline
\end{tabular}


Emmet Keefe ${ }^{9}$ revisou casos de hepatite aguda A em populações com doença hepática crônica subjacente em epidemias que ocorreram em Formosa, em 1988, e nos Estados Unidos, de 1983 a 1988. Revisou também séries menores e relatos de casos. Segundo esse autor, a literatura sugere que HVA, superposta em infecção crônica B, é associada com alterações laboratoriais e doença mais grave, incluindo a forma fulminante, e também com uma maior taxa de mortalidade nesses pacientes. Além disso, parece haver também uma maior taxa de mortalidade relacionada à HVA em pacientes com outros tipos de hepatopatia crônica ${ }^{9,17}$.

A análise dos dados epidemiológicos reportados ao CDC (Centers for Disease Control and Prevention), nos Estados Unidos, de 1983 a 1988, revelou uma taxa de mortalidade, por hepatite $\mathrm{A}$, de $11,7 \%$ nos pacientes com diagnóstico de hepatite crônica $B$, e de 4,6\% nos pacientes com outras hepatopatias crônicas pré-existentes. As fatalidades ocorreram principalmente na população mais velha (72\% das mortes acima dos 49 anos). Essas taxas são, respectivamente, 58 e 23 vezes mais altas do que na população sem doença hepática crônica subjacente ${ }^{9,10,18}$.

Todos esses estudos apresentam problemas metodológicos que limitam a sua comparação e generalização. Entretanto, parece haver evidências de que há um risco aumentado de morte em pacientes com hepatites crônicas quando contraem HVA, principalmente nos estudos populacionais de levantamentos de óbitos ${ }^{10}$. A associação entre morte por hepatite A fulminante e doença hepática crônica foi demonstrada em um estudo recente, baseado nos atestados de óbito de 1981 a 1997, do Centro Nacional de Estatísticas de Saúde dos EUA ${ }^{18}$. Nesse estudo de casos e controles, $63 \%$ das mortes por HVA incluíam menção de doença hepática crônica no atestado de óbito, comparado com 8 a 11\% das mortes por outras causas gastrintestinais.

Considerando-se a epidemia de Formosa, em 1988, em que mais de 300 mil pessoas foram contaminadas por frutos do mar com vírus $\mathrm{A}$, a taxa caso-mortalidade, nos indivíduos portadores de vírus $\mathrm{B}$, foi de $0,05 \%$, o que é 5,6 vezes mais alta do que em pacientes sem hepatopatia crônica B. Nessa epidemia, a população atingida era mais jovem, o que talvez explique a menor mortalidade ${ }^{9}$.

Sandro Vento e cols. ${ }^{2}$ estudaram prospectivamente, durante 7 anos, 595 adultos portadores de hepatite crônica $\mathrm{B}$ ou C, previamente soronegativos para HVA. Vinte e sete pacientes (10 com HVB e 17 com HVC) apresentaram hepatite aguda A nesse período. Hepatite fulminante ocorreu em 7 pacientes do grupo da hepatite $\mathrm{C}$, sendo que apenas 1 indivíduo não morreu. Eles concluíram que os pacientes com hepatite $\mathrm{C}$ crônica têm um risco substancial de morte, quando associada à superinfecção pelo vírus $\mathrm{A}$.

Evangelos Akriviadis e Redeker ${ }^{19}$ descreveram 4 pacientes adultos que morreram após desenvolver HVA. Os 4, na necropsia, mostravam doença hepática crônica subjacente.

Ao contrário dessas e de outras séries de indivíduos, alguns autores não indicam diferenças no prognóstico de pacientes com hepatopatia crônica quando adquirem o vírus $\mathrm{A}$, principalmente naqueles mais jovens ${ }^{16}$. Viola $\mathrm{e}$ cols. ${ }^{20} \mathrm{e}$ Zachoval e cols. ${ }^{21}$ não encontraram diferenças nos níveis de bilirrubina, transaminases e nem na evolução de pacientes portadores crônicos do vírus $\mathrm{B}$, com infecção aguda por HVA. Ambos sugerem que possa haver alguma interferência viral, pois notaram diminuição nos títulos de HBsAg dos pacientes durante a HVA. Helbling e Kammerlander ${ }^{22}$, na Suíça, não encontraram associação entre hepatite fulminante A e hepatite crônica B ou C subjacente.

Nenhuma morte ocorreu em mais de 6.000 casos de HVA notificados de 1992 a 1996, através do sistema SEIEVA de notificação de hepatite viral aguda, na Itália ${ }^{23}$. Entre esses casos de HVA, havia 179 indivíduos com hepatite crônica B ou C.

Desde 1996, o ACIP (Comitê Consultivo sobre Práticas de Imunização do CDC dos Estados Unidos) vem recomendando a imunização de todos os hepatopatas crônicos contra HVA $5,6,24$. A recomendação é baseada na presunção de que se um indivíduo já apresenta uma doença crônica do fígado, uma lesão hepática adicional pode vir a ser precariamente controlada.

Atualmente, os guias de imunização claramente estabelecem que pacientes com doença crônica do fígado devem receber a vacina da $\mathrm{HVA}^{5,6}$. A Organização Mundial da Saúde e o CDC recomendam a imunização contra HVA nas pessoas portadoras de hepatopatia crônica, devido à taxa aumentada de fatalidades para aqueles que adquirem a infecção pelo vírus $\mathrm{A}^{5,6,24}$. A Academia Americana de Pediatria, desde 1996, indica a vacinação contra HVA em pacientes hepatopatas crônicos ${ }^{25}$, assim como a Sociedade Brasileira de Pediatria ${ }^{26,27}$.

Para que se possa indicar a vacina contra HVA em hepatopatas crônicos, assim como em qualquer outro grupo de indivíduos, necessitamos conhecer a prevalência da infecção naquele grupo específico ${ }^{28,29}$.

$\mathrm{Na}$ amostra estudada, de 59 pacientes pediátricos, portadores de hepatopatias crônicas de diferentes etiologias, houve uma positividade de $24 \%$ para o anticorpo antiHVA. Essa porcentagem é baixa, considerando-se a idade e o fato de que a maioria do grupo tem renda familiar menor do que $\mathrm{R} \$ 500,00$. Houve um leve aumento na porcentagem da positividade do anti-HVA em relação à idade, porém sem significância estatística. Além disso, parece haver também uma tendência de os pacientes positivos serem aqueles com menores rendas familiares. Mas a diferença não é significante do ponto de vista estatístico, decorrente também do pequeno número de pacientes. A relação com o diagnóstico é mais provavelmente explicada pela idade do que pela etiologia. Assim, os pacientes com hepatite autoimune, com a maior média de idade, apresentaram uma maior positividade de anti-HVA. Apesar do grupo da atresia biliar ser maior, apresentou menor positividade, pois a média de idade é menor do que os outros 2 grupos ( 3,9 anos $\mathrm{x} 11,8$ na hepatite auto-imune x 6,2 anos na cirrose criptogênica). 
Koçak e colaboradores ${ }^{28}$, da Turquia, encontraram uma taxa de soropositividade para o anti-HVA de $44 \%$ entre 403 crianças e adolescentes portadores de hepatopatias crônicas, com idades entre 1,5 e 20 anos. Entre esses pacientes, 267 eram portadores de hepatite crônica B, e 136 de outras doenças crônicas do fígado. As 140 crianças que não eram portadoras de hepatopatias apresentaram $31 \%$ de soropositividade para o anti-HVA. A prevalência de anticorpos antiHVA aumentou com a idade: $16 \%$ nas crianças menores do que 5 anos, 35,5\% naquelas entre 6 e 10 anos, 59,5\% nas entre 11 e 15 anos, e $67 \%$ nos pacientes com mais de 15 anos de idade.

Diago e colaboradores ${ }^{29}$, na Espanha, estudaram a prevalência de anti-HVA em 425 adultos portadores de hepatopatias ( $\mathrm{x}=40$ anos), e encontraram $75 \%$ de positividade, que variou de $20 \%$ aos 19 anos até $93 \%$, naqueles com mais de 40 anos de idade.

A redução da suscetibilidade de uma população, através da vacinação, pode eliminar doenças e suas complicações, mas atualizações periódicas dos dados soroepidemiológicos são necessárias para avaliar os níveis de imunização natural e identificar aqueles suscetíveis à infecção.

Vacinar pacientes com doenças crônicas do fígado, protegendo-os de lesão hepática adicional ocasionada pelo vírus A, parece ser uma decisão inteligente e acertada ${ }^{1,10,22}$. Este estudo mostra que a maioria dos pacientes pediátricos (76\%) com hepatopatias crônicas é anti-HVA negativo, o que os torna suscetíveis à infecção pelo vírus $\mathrm{A}$ e suas potenciais complicações. Deve-se também levar em conta que, em países como o Brasil, o risco de se adquirir a infecção é muito grande, pois o vírus é endêmico e existem pessoas suscetíveis ${ }^{13,15}$.

Ainda não está bem estabelecido o esquema de imunização e nem as taxas de soroconversão para os pacientes com doença hepática crônica. Estudos de prevalência do anti-HVA, com posterior vacinação de hepatopatas crônicos, poderão colaborar no estabelecimento das normas de imunização para este grupo de indivíduos.

\section{Agradecimentos}

Agradecemos ao Laboratório Weinmann, pela realização dos exames anti-HVA, e à Vânia Hirakata, pela realização da análise estatística.

\section{Referências bibliográficas}

1. Sjogren MH. Prevention of acute liver disease in patients with chronic liver disease. Hepatology 1998;27:887-8.

2. Vento S, GarofanoT, Renzini C, Cainelli F, Casali F, Ghironzi G, et al. Fulminant hepatitis associated with hepatitis A virus superinfection in patients with chronic hepatitis C. N Engl J Med 1998;338:286-90.

3. Nebbia G, Giacchino R, Soncini R, Ramaccioni V, Timitilli A, Zanetti A. Hepatitis A vaccination in chronic carriers of hepatitis B virus. J Pediatr 1999;134:784-5.

4. Gunther M, Stark K, Neuhaus R, Reinke P, Schroder K, Bienzle U. Rapid decline of antibodies after hepatitis A immunization in liver and renal transplant recipients. Transplantation 2001;71:477-90.
5. MMWR. Prevention of hepatitis A through active and passive immunization: Recommendations of the Advisory Committee on Immunization Practices (ACIP). MMWR Morb Mortal Wkly Rep 45, 1996;RR-15:1-31.

6. MMWR. Prevention of hepatitis A through active and passive immunization: Recommendations of the Advisory Committee on Immunization Practices (ACIP). MMWR Morb Mortal Wkly Rep 48, 1999;RR-12,1-37.

7. Innis BL, Snitbhan R, Kunasol P, Laorakpongse T, Poopatanakool W, Kozik C, et al. Protection against hepatitis A by an inactivated vaccine. JAMA 1994;271:1328-34.

8. Werzberger A, Mensch B, Kuter B. A controlled trial of a formalininactivated hepatitis A vaccine in healthy children. N Engl J Med 1992;327:453-7.

9. Keeffe EB. Is hepatitis A more severe in patients with chronic hepatitis B and other chronic liver diseases? Am J Gastroenterol 1995;90:201-5.

10. Bell BP. Hepatitis A and hepatitis B vaccination of patients with chronic liver disease. Acta Gastroenterol Belg 2000;63:359-63.

11. Alter MJ, Mast TE. The epidemiology of viral hepatitis in the United States. Gastroenterol Clin North Am 1994;23:437-55.

12. Tapia-Conyer R, Santos JI, Cavalcanti AM, Urdaneta E, Rivera L, Manterola A, et al. Hepatitis A in Latin America: a changing epidemiologic pattern. Am J Trop Med Hyg 1999;61:825-9.

13. Ferreira CT, Barros FC, Silva GL, Pereira-Lima J. Soroepidemiologia da Hepatite A em dois grupos populacionais economicamente distintos de Porto Alegre. Gastroenterologia Endoscopia Digestiva 1996;15:85-90.

14. Gust ID. Epidemiological patterns of hepatitis A in different parts of the world. Vaccine 1992;10 (Suppl 1):56-58.

15. Costa-Clemens SA, Fonseca JC, Azevedo T, Cavalcanti AM, Silveira TR, Castilho MC, et al. Soroprevalência para hepatite A e hepatite B em 4 centros no Brasil. Rev Soc Bras Med Trop 2000;33:1-10.

16. Gust ID, Feinstone, SM. Hepatitis A. Prog Liver Dis 1990;9:371-8.

17. Keeffe EB, Iwarson S, McMahon BJ, Lindsay KL, Koff RS, Manns $\mathrm{M}$, et al. Safety and immunogenicity of hepatitis A vaccine in patients with chronic liver disease. Hepatology 1998;27:881-6.

18. Datta D. Association between deaths due to hepatitis A and chronic liver disease: United States, 1981 - 1997. Antiviral Ther 2000;5 (Suppl 1):79.

19. Akriviadis EA, Redeker AG. Fulminant hepatitis A in intravenous drug users with chronic liver disease. Ann Int Med 1989;110:838-9.

20. Viola LA, Barrison IG, Coleman JC, Murray-Lion IM. The clinical course of acute type A hepatitis in chronic HBsAg carriers-a report of 3 cases. Postgrad Med J 1982;58:80-8.

21. Zachoval R, Roggendorf M, Deinhardt F. Hepatitis A infection in chronic carriers of hepatitis B virus. Hepatology 1983;3:528-31.

22. Helbling B, Kammerlander R. Acute Hepatits A in patients with chronic hepatitis C. Ann Int Med 1999;131:314.

23. Mele A, Tosti ME, Stroffolini T. Hepatitis associated with hepatitis A superinfection in patients with chronic hepatitis C. N Engl J Med 1998;338:1771-3.

24. Centers for Disease Control. Recommended Childhood Immunization Schedule - United States, 2001. JAMA 2001;285:8 75-6.

25. American Academy of Pediatrics. Committee on Infectious Diseases. Prevention of Hepatitis A infection: guidelines for use of Hepatitis A vaccine and immune globulin. Pediatrics 1996;98:1207-15.

25. Sociedade Brasileira de Pediatria. Indicações - Guia de Adolescência: orientação para profissionais da área médica. Rio de Janeiro; 2000.

26. Sociedade Brasileira de Pediatria. Indicações de Vacinas. Disponível em: <http://www.sbp.com.br. Acessado em março de 2002.

27. Koçak N, Dinler G, Ozen H, Yuce A, Gurakan F. Prevalence of hepatitis A antibodies in children with chronic liver disease and other gastrointestinal diseases. Eur J Pediatr 1999;158:869-70.

28. Diago M, Lujan M, Garcia V, Cors R, Carbonell P, Tuset C, et al. Prevalence of anti-hepatitis A in patients with chronic liver disease. Gastroenterol Hepatol 1998;21:324-6. 